\title{
Methyl Group Effect on the Proton Affinity of Methylated Acetophenones Studied by Two Mass Spectrometric Techniques ${ }^{\dagger}$
}

\author{
A. Kukol, F. Strehle, G. Thielking and H.-Fr. Grützmacher* \\ Fakultät für Chemie, Universität Bielefeld, Postfach 1001 31, D-33501 Bielefeld 1, Germany
}

\begin{abstract}
The proton affinities $(P A)$ of all isomeric dimethylacetophenones were determined using the 'kinetic method' with a tandem mass spectrometer and by measurements of the proton transfer equilibrium in the gas phase by Fourier transform ion cyclotron resonance mass spectrometry. For both methods acetophenone and $p$-methylacetophenone, with well known $P A$ values, were used as reference bases. Both methods yielded identical $P A$ values for all dimethylacetophenones. The $P A$ of the dimethylacetophenones were in a narrow range between 872 and $880 \mathrm{~kJ}$ mol $^{-1}$ except for 2,6-dimethylacetophenone, for which a $P A$ of $856 \mathrm{~kJ} \mathrm{~mol}^{-1}$ was found. The results are discussed in terms of possible mesomeric structures stabilizing the positive charge with regard to the substitution pattern in the phenyl ring. Obviously, the significant smaller $P A$ of 2,6-dimethylacetophenone compared with the other isomers is due to the distortion of the conjugation of the $\mathrm{C}-\mathrm{O}$ double bond with the aromatic $\pi$ system.
\end{abstract}

\section{INTRODUCTION}

Proton affinity $(P A)$ is a fundamental molecular property and of considerable interest for the understanding of the relationship between the structure and reactivity of molecules and ions in the gas phase. In the last few years, an increasing number of publications has appeared dealing with the experimental determination of the gas-phase basicity $(G B)$ of simple organic compounds ${ }^{1-7}$ and of large, non-volatile biological molecules. $^{8-10}$ Depending on the properties of the substance to be studied, different mass spectrometric methods have been developed to determine $P A$ values. The application of the measurement of rate constants of ion-molecule reactions in the gas phase to determine the equilibrium constant of the following protontransfer was first introduced by Kebarle and coworkers: ${ }^{11}$

$$
\mathrm{AH}^{+}+\mathrm{B} \rightleftharpoons \mathrm{A}+\mathrm{BH}^{+}
$$

The change in the Gibb's free energy $(\Delta G)$ of the equilibrium is given by the well known relationship

$$
\Delta G=-R T \ln K
$$

where $R$ is the universal gas constant, $T$ is the absolute temperature of the system and $K$ is the equilibrium constant at this temperature:

$$
K=\frac{\left[\mathrm{BH}^{+}\right][\mathrm{A}]}{\left[\mathrm{AH}^{+}\right][\mathrm{B}]}
$$

The difference between the gas-phase basicities of compounds $\mathrm{A}$ and $\mathrm{B}$ is easily determined by measuring the

† Dedicated to Professor J. L. Holmes in honour of his great contribution to Organic Mass Spectrometry and to gas-phase ion chemistry. intensity ratio of the signals corresponding to the two protonated species combined with the measurement of the partial pressure ratio of the neutral species in a high-pressure mass spectrometer, ${ }^{2}$ in a Fourier transform ion cyclotron resonance (FT-ICR) spectrometer ${ }^{5}$ or in an ion-trap mass spectrometer. ${ }^{12}$ This method gives reliable data for the $P A$, but it requires volatile compounds of high purity.

Another method first proposed by Cooks and Kruger $^{13}$ is the so-called 'kinetic method', which allows less pure and non-volatile substances also to be investigated. For this method, proton-bound heterodimers $\left\{\mathrm{A} \cdot \mathrm{H}^{+} \cdots \mathbf{B}\right\}$ are generated in a chemical ionization (CI) ion source of a tandem mass spectrometer. Using approximations based on the thermal kinetics of competitive reactions and applying this to the rate constants of the metastable dissociations of the proton-bound heterodimer $\left\{\mathrm{A} \cdots \mathrm{H}^{+} \cdots \mathrm{B}\right\}$, the equation

$$
\ln \left(k_{1} / k_{2}\right)=\Delta P A / R T
$$

is obtained, where $k_{1}$ and $k_{2}$ are the rate constants of the competitive fragmentations of the cluster ion:

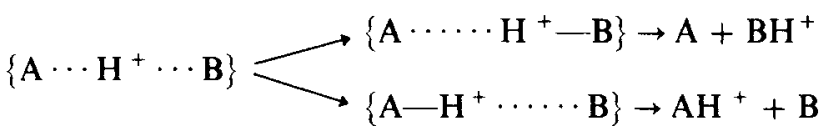

After forming the heterodimer in a high-pressure ion source using conditions for $\mathrm{CI}$, the dimer is selected by its specific mass to charge ratio in the first stage of a tandem mass spectrometer. The metastable dissociation of the cluster leads to intense signals of the two protonated monomers. The intensity ratio of the peaks of these protonated monomers corresponds to the ratio of 
Table 1. Proton affinities of the monomethylated isomeric benzaldehydes ${ }^{17}$ and acetophenones, ${ }^{20,21}$ determined using the equilibrium method

\begin{tabular}{cccc} 
Compound & \multicolumn{3}{c}{$P$ A $\left(\mathrm{kJ} \mathrm{mol}^{-1}\right)$} \\
& o-Methyl- & $m$-Methyl- & $\rho$-Methyl- \\
Monomethylated benzaldehyde & 851 & 848 & 854 \\
Monomethylated acetophenone & 864 & 862 & 873 \\
\hline
\end{tabular}

the rate constants of the competing dissociations [Eqn (5)]:

$$
\ln \left(k_{2} / k_{1}\right)=\ln \left(\left[\mathrm{AH}^{+}\right] /\left[\mathrm{BH}^{+}\right]\right)
$$

Combining Eqns (4) and (6), one obtains

$$
\ln \left(\left[\mathrm{AH}^{+}\right] /\left[\mathrm{BH}^{+}\right]\right)=\Delta P A / R T
$$

Details of the theoretical basis can be found in the literature cited in Refs 14 and 15 .

The $P A$ values of differently substituted acetophenones, ${ }^{4.16}$ benzaldehydes, ${ }^{17}$ benzamides ${ }^{18}$ and methyl benzoates ${ }^{19}$ have been determined and the effects of substituents on the $P A$ have been discussed. The results show that there is a strong resonance interaction between electron-donating substituents at the para position to the $O$-protonated carbonyl group. However, the effect of ortho substituents is less clear because of possible steric effects on the coplanar orientation of the protonated carbonyl group and the phenyl ring which is a prerequisite for a strong resonance interaction. In Table 1 the $P A$ values of monomethyl-substituted benzaldehydes ${ }^{17}$ and acetophenones ${ }^{20,21}$ are given. For the methyl substituent the $P A$ of the para-substituted aldehydes and ketones are affected by stabilization of the protonated carbonyl group by the methyl substituent across the benzene ring. In principle, methyl substitution at the ortho position can stabilize the positive charge of the protonated carbonyl group similarly, but a distinct effect is observed only for benzaldehydes. In the case of monomethylacetophenones, the methyl effect on the $P A$ is nearly identical for the ortho and meta positions. This is a clear indication of some steric hindrance of the conjugation between the carbonyl double bond and the $\pi$ system of the phenyl ring by one $o$ methyl group. The aim of this work was to investigate<smiles>Cc1cccc(C(C)(C)C)c1C</smiles>

1<smiles>Cc1ccc(C(=O)C(C)(C)C)c(C)c1</smiles>

4<smiles>Cc1ccc(C)c(C(=O)C(F)(F)F)c1</smiles>

2

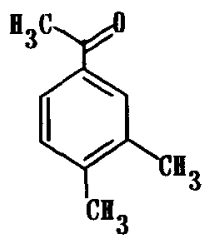

5

Figure 1. Substitution pattern of the dimethylacetophenones. investigated. quantitatively the antagonistic effect due to mesomeric stabilization and steric hindrance of the methyl group by the determination of the $P A$ values of the isomeric dimethylacetophenones 1-6 (Fig. 1). The measurements were performed by equilibrium techniques using FT-ICR mass spectrometry and by the kinetic method using tandem mass spectrometry. This allows also a direct comparison of these two very different experimental concepts of $P A$ determination for one class of compounds.

\section{EXPERIMENTAL}

Compounds 1, 4, 5 and 6 were synthesized from commercially available dimethylanilines. The diazotization of the anilines followed by the Sandmeyer reaction using standard procedures of organic chemistry leads to the corresponding nitriles from which the acetophenones are obtained by a Grignard reaction with methylmagnesium iodide. Acetophenones 2 and 3 were obtained by Friedel-Crafts acylation of the corresponding xylenes.

The structure of the substances were verified by ${ }^{1} \mathrm{H}$ NMR and $70 \mathrm{eV}$ electron impact mass spectrometry. The purity was monitored by means of appropriate chromatographic techniques.

FT-ICR mass spectrometry was achieved using a Bruker Spectrospin CMS 47X mass spectrometer equipped with a $4.7 \mathrm{~T}$ superconducting magnet, a cylindrical cell of $6 \mathrm{~cm}$ length and $6 \mathrm{~cm}$ diameter and a 24 bit, $128 \mathrm{~K}$ Aspect 3000 computer. The particular compound under study and the reference substance were introduced by two separate needle valves controlling the partial pressure of each component. The total pressure within the ICR cell was adjusted to between $1 \times 10^{7}$ and $3 \times 10^{-7} \operatorname{mbar}(1 \mathrm{mbar}=100 \mathrm{~Pa})$, read uncorrected from the ion gauge in front of the highvacuum turbo pump. The ions were generated by internal ionization using a 3.0-3.5 A filament current and $70 \mathrm{eV}$. Under these conditions the ions $\mathrm{AH}^{+}$and $\mathrm{BH}^{+}$ were formed by 'self-CI' and then isolated using the usual ejection technique. After ejection of either $\mathrm{AH}^{+}$or $\mathrm{BH}^{+}$the ions were allowed to react with the neutral species. An equilibrium indicated by a constant $\mathrm{AH}^{+} / \mathrm{BH}^{+}$ratio was achieved for every $\mathrm{A}-\mathrm{B}$ pair reported in this paper within $10 \mathrm{~s}$. The $\mathrm{AH}^{+} / \mathrm{BH}^{+}$ratio and the corresponding ratio of the partial pressure of the neutral species were then used to calculate $G B(\mathrm{~A})$ according to Eqns (2) and (3). The equilibrium temperature [Eqn (2)] was determined by comparison of the reaction rate for $\mathrm{CH}_{4}^{+\cdot}+\mathrm{CH}_{4}$ for external ionization and for the ionization of $\mathrm{CH}_{4}$ using the internal filament. $^{22}$ Thus, the temperature inside the ICR cell of the Bruker Spectrospin CMS $47 \mathrm{X}$ instrument was estimated to be $\sim 320 \mathrm{~K}$.

The metastable ion fragmentations of the protonbound heterodimer clusters were investigated using a VG AutoSpec mass spectrometer with EBE geometry. The cluster ions were generated in a $\mathrm{CI}$ ion source, adjusting the pressure so as to be sufficient for self-CI and the dimerization of the protonated neutral species. 
The cluster ions were extracted at $8 \mathrm{kV}$, selected by the first two sectors (EB) and then allowed to dissociate spontaneously in the third field-free region without the use of a collision gas (mass-analysed ion kinetic energy (MIKE) spectrometry). The mass resolution of the parent ion was 4000 . The MIKE spectrum was achieved by scanning the second electrostatic analyser. The number of scans accumulated was $10-20$ to improve the signal-to-noise ratio. In the case of identical half-widths of the peaks of $\mathrm{AH}^{+}$and $\mathrm{BH}^{+}$, the corresponding ion abundances can be calculated by using the peak height only. This was true for all compounds investigated here. Inserting the $\mathrm{AH}^{+} / \mathrm{BH}^{+}$peak intensity ratio in Eqns (6) and (4), the $P A$ of $A$ is obtained. The temperature required in Eqn (4), which is the effective temperature ${ }^{14}$ of the dimer ions undergoing unimolecular dissociation in the selected region, was derived by measuring the dissociation proton-blound heterodimers formed by each of the monomethylated acetophenones and acetophenone, resulting in a value of $T \sim 400 \mathrm{~K}$.

\section{RESULTS AND DISCUSSION}

4-Methylacetophenone (7) was used as a reference to determine the gas-phase basicity $(G B)$ of the dimethylacetophenones 1-5 and acetophenone (8) was used as a reference for the 2,6-isomer 6 . The $G B$ of 7 and 8 at $298 \mathrm{~K}$ were taken from the literature: ${ }^{23} \mathrm{~GB}(7)=840.5$

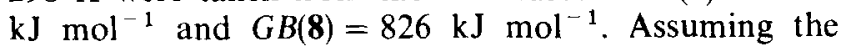
entropy change due to the protonation equilibrium arises mainly from the entropy change of the proton $\left(\Delta S=-108.7 \mathrm{JK}^{-1} \mathrm{~mol}^{-1}\right),{ }^{23,25}$ values of $P A(7)=873$ $\mathrm{kJ} \mathrm{mol}^{-1}$ and $P A(8)=859 \mathrm{~kJ} \mathrm{~mol}^{-1}$ are obtained. In Table 2 the results of the determination of the $P A$ values for the dimethylated acetophenones 1-6 are given.

The $P A$ values obtained from both the equilibrium method and the kinetic method are in excellent agreement. Because the equilibrim method is not limited by conditions concerning the chemical structure and functionality of the species $A$ and $B$, one may expect the relative $G B$ obtained with the classical thermodynamic equilibrium method to be more reliable if competitive dimerization reactions ${ }^{1}$ are avoided by low pressure in the FT-ICR cell. In contrast, the kinetic method compares the unimolecular fragmentation rates $\left(k_{1}, k_{2}\right)$ of

Table 2. Proton affinities of all isomeric dimethylacetophenones obtained using the equilibrium and kinetic methods $^{\mathrm{a}}$

\begin{tabular}{cccc} 
& & \multicolumn{2}{c}{$P A\left(\mathrm{~kJ} \mathrm{~mol}^{-1}\right)$} \\
No. & Compound & Equilibrium method & Kinetic method \\
1 & 2,3-Dimethylacetophenone & 872 & 871 \\
2 & 2,5-Dimethylacetophenone & 871 & 872 \\
3 & 3,5-Dimethylacetophenone & 872 & 875 \\
4 & 2,4-Dimethylacetophenone & 880 & 878 \\
5 & 3,4-Dimethylacetophenone & 879 & 879 \\
6 & 2,6-Dimethylacetophenone & 855 & 857
\end{tabular}

a The maximum experimental error is estimated to be $2 \mathrm{~kJ} \mathrm{~mol}^{-1}$ for both methods. two competitive processes [Eqn (5)] far from the thermal equilibrium. This is also indicated by the effective temperature $T$, found to be $\sim 400 \mathrm{~K}$ for the ion structures and time-scale studied here. An important approximation for the validity of Eqn (4) is the assumpton that the activation barrier for the reverse reaction of Eqn (5) is negligible, which is the case in the absence of any structural rearrangements in the protonated cluster. However, if the cluster rearranges, forming covalent bonds, the mechanisms for the formation of $\mathrm{AH}^{+}$and $\mathrm{BH}^{+}$are unknown and the entropic factors for the two reactions probably differ significantly, leading to wrong $P A$ values. As pointed out by Cooks and coworkers, ${ }^{14,15}$ Eqn (4) gives reliable results only if the partition functions of the two activated complexes and of the product ions $\mathrm{AH}^{+}$and $\mathrm{BH}^{+}$are identical. This is correct only for proton-bound homodimers $\left\{A \cdots H^{+} \cdots A\right\}$, but should be sufficiently approximated by a pair of chemically very similar compounds $A$ and $B$. The reference bases 7 and 8 and compounds 1-6 are similar aromatic ketones, and the bonding centre of the proton in the proton-bound heterodimers is probably always the carbonyl oxygen, resulting in identical vibrational frequencies of the $\mathrm{A}-\mathrm{H}$ and $\mathrm{B}-\mathrm{H}$ stretching mode. Thus, following Cooks and coworkers' application of the statistical rate theory to the kinetic approach, ${ }^{15}$ one may assume that the vibrational partition functions of the two protonated species are equal, and that the simplified Eqn (4) holds. Finally, the $P A$ differences between the bases 7 and 8 and compounds $1-6$ are not larger than the $7 \mathrm{~kJ} \mathrm{~mol}^{-1}$ which is required to guarantee good accuracy of the two methods.

A comparison of the $P A$ values of the dimethylacetophenones 1-6 in Table 2 shows that those of the 2,3-, 2,5- and 3,5-dimethylated isomers are identical, within the limits of the experimental error. Thus, methyl substituents at the ortho and meta positions contribute similarly to the $P A$ of dimethylacetophenones and to the stabilization of the positive charge of a protonated carbonyl group on the aromatic ring. Note that this has already been observed in the case of the monomethylated acetophenones ${ }^{20.21}$ (Table 1). Clearly, the resonance stabilization of the positive charge by an $o-$ methyl group is partially compensated for by steric hindrance of the conjugation between the protonated carbonyl group and the aromatic $\pi$-electron system by twisting the carbonyl group out of the plane of the phenyl ring. This effect, however, is not enhanced by 'buttressing' the o-methyl group by a second adjacent methyl substituent, as indicated by the identical $P A$ values of 2,3- and 2,5-dimethylacetophenone.

In line with a similar methyl group effect at the ortho and meta positions of acetophenone is the observation of identical $P A$ values for 2,4- and 3,4-dimethylacetophenones. In the case of the monomethyl derivatives a $p$-methyl substituent increases the $P A$ of acetophenone by $8 \mathrm{~kJ} \mathrm{~mol}^{-1}$ with respect to the other isomers, and the same increase in the $P A$ is observed for dimethylacetophenones containing a $p$-methyl group. Hence, although the electronic interaction between the p-methyl substituent and the protonated carbonyl group across the benzene ring stabilizes the coplanar orientation of the latter group the steric interaction of 
the o-methyl group overrides the para contribution to the same extent as the effect of a $m$-methyl group.

As would be expected from the distinct steric effect of one o-methyl substituent on the coplanarity of the protonated acetophenone, the presence of two o-methyl groups in 2,6-dimethylacetophenone leads to an extreme situation. The $P A$ of $856 \mathrm{~kJ} \mathrm{~mol}^{-1}$ of this isomer 6 is very small and, in fact, smaller than the $P A$ of the unsubstituted acetophenone. Isopropyl methyl ketone has a carbon skeleton at the keto group related to 6 , and the $P A$ of this saturated aliphatic ketone without any resonance stabilization of the protonated carbonyl group is $851 \mathrm{~kJ} \mathrm{~mol}^{-1} .{ }^{20}$ Thus, it appears that the (protonated) carbonyl group of 6 is twisted into an orthogonal orientation to the aromatic ring so that the stabilization of the positive charge in the protonated isomer 6 occurs by inductive and polarization effects typical for saturated aliphatic ketones. It will be of interest to see whether the resonance between the protonated carbonyl group and the aromatic ring can be restored by the much stronger electronic effects of electron-donating polar substituents in the para position of 2,6-dimethylacetophenone. A study of the protonation of these acetophenones is in progress. ${ }^{26}$ However, the methyl group effect on the $P A$ of the dimethylated acetophenones shows already that the phenomenon of the steric inhibition of resonance, well known from liquid-phase chemistry, ${ }^{27}$ can also be understood in terms of the thermochemical properties of isolated gas-phase molecules and ions.

\section{Acknowledgement}

The financial support of this work by the Deutsche Forschungsgemeinschaft and additional support by the Fonds der Chemischen Industrie is gratefully acknowledged.

\section{REFERENCES}

1. M. T. Bowers (Ed.), Gas Phase Ion Chemistry, Vol. II. Academic Press, New York (1979).

2. P. Kebarle, Annu. Rev. Phys. Chem. 28, 445 (1977)

3. J. Bartmess, J. A. Scott and R. T. Mclver, J. Am. Chem. Soc 101, 606 (1979).

4. M. Meot-Ner (Mautner), E. P. Hunter and F. H. Field, J. Am Chem Soc. 101, 686 (1979).

5. R. Wolf and H. F. Grützmacher, New J. Chem. 14, 379 (1990).

6. H. H. Büker and H. F. Grützmacher, Int. J. Mass Spectrom Ion Processes 109, 95 (1991).

7. M. Meot-Ner (Mautner) and L. W. Sieck, J. Am. Chem. Soc 113, 4448 (1991).

8. G. Bojesen, J. Am. Chem Soc. 109, 5557 (1987).

9. K. Isa, T. Omote and M. Amaya, Org. Mass Spectrom. 25, 620 (1990).

10. Z. Wu and C. Fenselau, Rapid Commun. Mass Spectrom. 6, 403 (1992).

11. A. M. Hogg, R. N. Haynes and P. Kebarle, J. Am. Chem. Soc 88, 28 (1966).

12. B. D. Nourse and R. G. Cooks, Int. J. Mass Spectrom. Ion Processes 106, 249 (1991).

13. R. G. Cooks and T. L. Kruger, J. Am. Chem Soc. 99, 1279 (1977).

14. S. A. Mclucky, D. Cameron and R. G. Cooks, J. Am. Chem. Soc. 103, 1313 (1981).
15. T. K. Majumdar, F. Clairet, J.-C. Tabet and R. G. Cooks, J Am. Chem. Soc. 114, 2897 (1992).

16. M. Mishima, M. Fujio and Y. Tsuno, Tetrahedron Lett. 27, 939 (1986)

17. P. D. J. Anderson, M. T. Fernandez and R. S. Mason, presented at the XIXth Annual Meeting of the BMSS, St. Andrews, Scotland (1992).

18. A. Mersch and H. F. Grützmacher, to be published.

19. M. Mishima, M. Fujio and Y. Tsuno, Tetradhedron Lett. 27. $951(1986)$

20. S. G. Lias, J. E. Bartmess, J. F. Liebman, J. L. Holmes, R. D Levin and W. G. Mallard, J. Phys. Ref. Data 17, 1 (1988)

21. G. Thielking, PhD Thesis, Universität Bielefeld (1991).

22. W. F. Bailey and A. S. Monahan, J. Chem. Educ. 55, 489 (1987).

23. S. G. Lias, J. F. Liebman and R. D. Levin, J. Phys. Ref. Data 13, 695 (1984)

24. D. Thölmann, PhD Thesis, Universität Bielefeld (1992).

25. J. B. Cumming and P. Kebarle, Can. J. Chem. 56, 1 (1978).

26. H. Lustinetz, F. Strehle and H. F. Grützmacher, to be published.

27. P. Desiongchamps, Stereoelectronic Effects in Organic Chemistry. Pergamon Press Oxford (1983). 